\title{
Update on the diagnostic value and safety of stereotactic biopsy for pediatric brainstem tumors: a systematic review and meta-analysis of 735 cases
}

\author{
*Christina Hamisch, MD, ${ }^{1,2}$ Philipp Kickingereder, MD, ${ }^{3,4}$ Matthias Fischer, MD, ${ }^{5}$ \\ Thorsten Simon, MD, ${ }^{5}$ and Maximilian I. Ruge, MD ${ }^{1,4}$ \\ Departments of ${ }^{1}$ General Neurosurgery, ${ }^{3}$ Stereotaxy and Functional Neurosurgery, and ${ }^{5}$ Pediatric Hematology and Oncology, \\ University Hospital of Cologne; ${ }^{2}$ Center of Integrated Oncology (CIO), University of Cologne; and ${ }^{4}$ Department of Neuroradiology, \\ University of Heidelberg Medical Center, Heidelberg, Germany
}

\begin{abstract}
OBJECTIVE Recent studies have shed light on the molecular makeup of diffuse intrinsic pontine gliomas and led to the identification of potential treatment targets for these lesions, which account for the majority of pediatric brainstem tumors (pedBSTs). Therefore, stereotactic biopsy-driven molecular characterization of pedBSTs may become an important prerequisite for the management of these fatal brain tumors. The authors conducted a systemic review and meta-analysis to precisely determine the safety and diagnostic success of stereotactic biopsy of pedBSTs.
\end{abstract}

METHODS A systematic search of PubMed, EMBASE, and the Web of Science yielded 944 potentially eligible abstracts. Meta-analysis was conducted on 18 studies (including the authors' own institutional series), describing a total of 735 biopsy procedures for pedBSTs. The primary outcome measures were diagnostic success and procedure-related complications. Pooled estimates were calculated based on the Freeman-Tukey double-arcsine transformation and DerSimonian-Laird random-effects model. Heterogeneity, sensitivity, and meta-regression analyses were also conducted.

RESULTS The weighted average proportions across the analyzed studies were $96.1 \%(95 \% \mathrm{Cl} 93.5 \%-98.1 \%)$ for diagnostic success, $6.7 \%(95 \% \mathrm{Cl} 4.2 \%-9.6 \%)$ for overall morbidity, $0.6 \%(95 \% \mathrm{Cl} 0.2 \%-1.4 \%)$ for permanent morbidity, and $0.6 \%(95 \% \mathrm{Cl} 0.2 \%-1.3 \%)$ for mortality. Subgroup analyses at the study level identified no significant correlation between the outcome measures and the distribution of the chosen biopsy trajectories (transfrontal vs transcerebellar), age, year of publication, or the number of biopsy procedures annually performed in each center.

CONCLUSION Stereotactic biopsy of pedBSTs is safe and allows successful tissue sampling as a prerequisite for the molecular characterization and the identification of potentially druggable targets toward more individualized treatment concepts to improve the outcome for children harboring such lesions.

https://thejns.org/doi/abs/10.3171/2017.2.PEDS1665

KEY WORDS pediatric brainstem tumors; diagnostic yield; meta-analysis; procedural complications; stereotactic biopsy; DIPG; oncology

$\Delta$ LTHOUGH brainstem tumors are extremely rare in adults, they comprise approximately $10 \%-20 \%$ of all pediatric brain tumors. ${ }^{26}$ Diffuse intrinsic pontine gliomas (DIPGs), the most common subtype of pediatric brainstem tumors (pedBSTs), are associated with an invariably fatal prognosis, and fewer than $10 \%$ of children with these tumors survive 2 years from diagnosis. ${ }^{34}$ The role of stereotactic biopsy for patients with pedBSTs in general, and DIPG specifically, has been controversial. In 1993, Albright et al. reported the results of the Children's

ABBREVIATIONS AANS = American Association of Neurological Surgeons; $\mathrm{ASCO}=$ American Society of Clinical Oncology; $\mathrm{BST}=$ brainstem tumor; $\mathrm{CNS}=\mathrm{Congress}$ of Neurological Surgeons; DIPG = diffuse intrinsic pontine glioma; GMS = German Medical Science; IQR = interquartile range; pedBST = pediatric BST.

SUBMITTED January 31, 2016. ACCEPTED February 16, 2017.

INCLUDE WHEN CITING Published online June 16, 2017; DOI: 10.3171/2017.2.PEDS1665.

* Drs. Hamisch and Kickingereder contributed equally to this work. 
Cancer Group DIPG study and concluded that MRI was adequate in making the diagnosis, since tissue sampling did not alter the treatment or impact outcome. ${ }^{2}$ Based on this conclusion and the presumed risk profile of stereotactic biopsy procedures in the brainstem, referring to studies reporting transient or permanent morbidity rates of up to $28 \%$ and $9 \%$, respectively, $, 5,7$ and mortality rates of up to $4 \%,{ }^{31}$ tissue sampling has been rarely performed. Thus, very little information on the molecular biology of DIPG has been available until very recently, when collaborative efforts allowed for the collect of a critical number of specimens, primarily derived from autopsy tissue, for molecular characterization of these tumors., ${ }^{412}$ These analyses unraveled a unique genetic makeup of DIPG that is distinct from other pediatric high-grade gliomas or adult high-grade gliomas. ${ }^{28}$ Moreover, DIPGs were found to constitute a heterogeneous entity with 3 molecularly distinct subgroups (H3-K27 M, silent, and MYCN) identified with whole-genome sequencing with methylation, expression, and copy number profiling. ${ }^{4}$ This complexity needs to be considered when designing new therapeutic approaches, since each subgroup potentially requires a different treatment to improve outcome for these children. ${ }^{4}$ Therefore, stereotactic biopsy-driven molecular characterization may become an important prerequisite for the management of these tumors. We conducted a systemic review and meta-analysis to precisely determine the safety and diagnostic success of stereotactic biopsy for pedBSTs.

\section{Methods}

\section{Search Strategy and Study Identification}

The study was conducted in accordance with Preferred Reporting Items for Systematic Reviews and Meta-Analyses (PRISMA) guidelines, ${ }^{18}$ no protocol for this metaanalysis has been published or registered. Appropriate studies were identified by searching the electronic databases PubMed, EMBASE, and Web of Science. Search terms included: "pediatric" or "paediatric" or "children" or "child" or "adolescent" in combination with "biopsy" or "biopsies" or "stereotactic" and in combination with "brainstem" or "brain stem" or "pons" or "pontine" or "mesencephalon" or "midbrain" or "medulla oblongata" or "posterior fossa" or "infratentorial" or "brain" or "intracranial." Searches were limited to human studies published in English from 1980 and 2015. Reference lists from publications retrieved were also examined to identify additional studies. The websites of the American Association of Neurological Surgeons (AANS; 1997-2010), the Congress of Neurological Surgeons (CNS; 1997-2012), the American Society of Clinical Oncology (ASCO; meeting abstracts, 2004-2012; educational books, 2002-2012), and German Medical Science (GMS; 2003-2012) were also searched for relevant abstracts that met our inclusion criteria. In addition, we also evaluated our own institutional series on patients undergoing stereotactic biopsy for the diagnosis of pedBSTs over the period of 1994 to 2012 and included the results in the present meta-analysis (Supplementary Table 1 encompasses detailed characteristic and procedural metrics, e.g., diagnostic yield, mor-
TABLE 1. Baseline characteristics of the 18 studies included in the present meta-analysis

\begin{tabular}{|c|c|}
\hline Characteristic & Value \\
\hline \multicolumn{2}{|l|}{ Study design, no. of studies } \\
\hline Prospective & 1 \\
\hline Retrospective & 17 \\
\hline \multicolumn{2}{|l|}{ No. of pts in the individual studies } \\
\hline Median & 24 \\
\hline Range & $10-130$ \\
\hline \multicolumn{2}{|c|}{$\begin{array}{l}\text { No. of biopsy procedures performed/yr in the } \\
\text { individual institutions }\end{array}$} \\
\hline Median & 3.1 \\
\hline Range & $1.4-10.0$ \\
\hline \multicolumn{2}{|l|}{ Sex, $\%$ of pts $\dagger$} \\
\hline Female & 48 \\
\hline Male & 52 \\
\hline \multicolumn{2}{|l|}{ Age in yrsł } \\
\hline Mean & 8.0 \\
\hline Range & $6.0-12.8$ \\
\hline \multicolumn{2}{|l|}{ Biopsy planning based on } \\
\hline CT only, no. of studies & 8 \\
\hline CT \&/or MRI, no. of studies & 8 \\
\hline Data not available, no. of studies & 2 \\
\hline \multicolumn{2}{|l|}{ Applied biopsy trajectory, \% of pts§ } \\
\hline \multicolumn{2}{|l|}{ Transfrontal approach } \\
\hline Median & 60.0 \\
\hline IQR & $6.7-87.7$ \\
\hline \multicolumn{2}{|l|}{ Transcerebellar approach } \\
\hline Median & 40.0 \\
\hline IQR & $12.2-93.3$ \\
\hline
\end{tabular}

Pts = patients

* Information available for $81 \%$ of patients (599 patients, 16 studies). $\dagger$ Information available for $87 \%$ of patients (637 patients, 14 studies).

$\ddagger$ Based on the measure of central tendency (mean or median) used in the individual studies. Information available for $79 \%$ of patients (577 patients, 15 studies).

$\S$ Information available for $67 \%$ of patients ( 495 patients, 15 studies).

bidity, mortality) of patients in the local dataset; treatment planning and surgical procedure were performed as described previously ${ }^{27}$ ). The institutional review board of the University Hospital Cologne approved this retrospective data evaluation, and the requirement for patient informed consent was waived.

\section{Selection Criteria}

Selection of abstracts for full review was conducted by 2 independent investigators (P.K. and M.I.R.) based on predefined inclusion and exclusion criteria. Studies were eligible if they reported original data on stereotactic biopsy of brainstem tumors (defined as tumors located in the midbrain, pons, or medulla oblongata) in pediatric patients (age $<21$ years) including details on procedure-related complications (morbidity and mortality) and diagnostic 


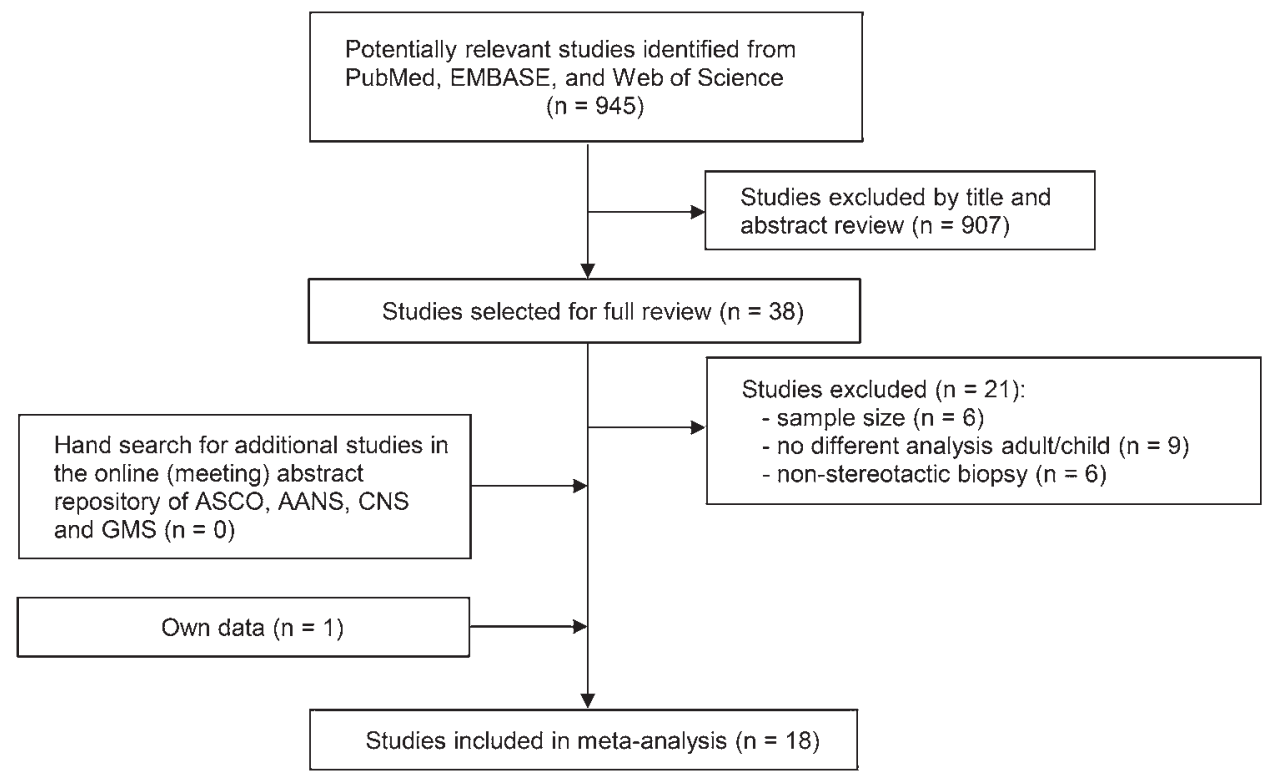

FIG. 1. Flowchart for search strategy and study selection; $n$ refers to number of studies.

success rates. Studies were excluded if they 1) presented a reanalysis of subpopulations already included in other studies; 2) reported on biopsy procedures unrestricted to a certain location, not separately addressing the outcome for the brainstem subpopulation; 3) reported on a group of less than 10 patients; or 4) were commentaries, technical notes, or review articles summarizing the results of previous series. Furthermore, we excluded those series reporting on nonstereotactic (open) biopsies, since this approach has generally been replaced by stereotactically guided biopsies for diagnosing BSTs. ${ }^{1,3,5,6,14,24,30}$ Each person reviewed the abstracts independently and generated a list of studies to retrieve for full-text review. Lists were then compared and discrepancies resolved by consensus.

\section{Data Extraction}

Extraction of data was independently performed by 2 authors (P.K. and M.I.R.) and subsequently verified between the 2 authors, with discrepancies settled through consensus discussion. The following details were extracted: treatment institution, year of publication, duration of study, number of patients, age (mean or median age of the study population), and the applied biopsy trajectory (transfrontal or transcerebellar). Furthermore, diagnostic success rates (number of patients with a valid histopathological diagnosis), detailed histopathological results, and procedure-related (overall and permanent) morbidity and mortality rates were extracted.

In a previously published paper, we also performed a literature review and meta-analysis of stereotactic biopsy for brainstem tumors..$^{15}$ The focus of that paper was the diagnostic value and safety of the method in the pediatric and adult population, and the research was limited to the period from 1980 to 2012. In this manuscript, we focused on the pediatric population only, updated the literature (1980-2015) and added data from our own case series.

\section{Statistical Analysis}

Statistical analysis was performed as described previously. ${ }^{15}$ In brief, study-specific proportions of outcome measures (diagnostic success, overall morbidity, permanent morbidity, and mortality) were transformed using the Freeman-Tukey variant of the arcsine square ${ }^{10}$ and then combined using DerSimonian-Laird random-effects meta-analyses. Study heterogeneity was assessed by the $\mathrm{I}^{2}$ statistic, with values of $25 \%, 50 \%$, and $75 \%$ representing mild, moderate, and severe inconsistency, respectively. ${ }^{13}$ Meta-regression was used for subgroup analysis at the study level. Evidence of publication bias was assessed with funnel plots. Sensitivity analysis was conducted by repeatedly calculating the effect size with 1 study omitted per iteration. All analyses were performed with Stata

TABLE 2. Pooled pathological diagnoses (of 735 patients) from the 18 studies included in the present meta-analysis

\begin{tabular}{crr}
\hline \multicolumn{1}{c}{ Pathology } & No. of Pts & $\%$ \\
\hline Neoplastic disease & 676 & 92.0 \\
\hline Glioma & 620 & 84.4 \\
\hline Low grade (WHO Grade I-II) & 206 & 28.0 \\
\hline High grade (WHO Grade III-IV) & 169 & 23.0 \\
\hline Information regarding WHO grade not provided & 245 & 33.3 \\
\hline PNET & 22 & 3.0 \\
\hline Ependymoma & 16 & 2.2 \\
\hline Other tumors & 18 & 2.4 \\
\hline Nonneoplastic disease & 33 & 4.5 \\
\hline Infectious disease & 16 & 2.2 \\
\hline Inflammatory disease & 3 & 0.4 \\
\hline Other nonneoplastic diseases & 14 & 1.9 \\
\hline Nondiagnostic biopsy procedure & 26 & 3.5 \\
\hline
\end{tabular}

PNET = primitive neuroectodermal tumor. 


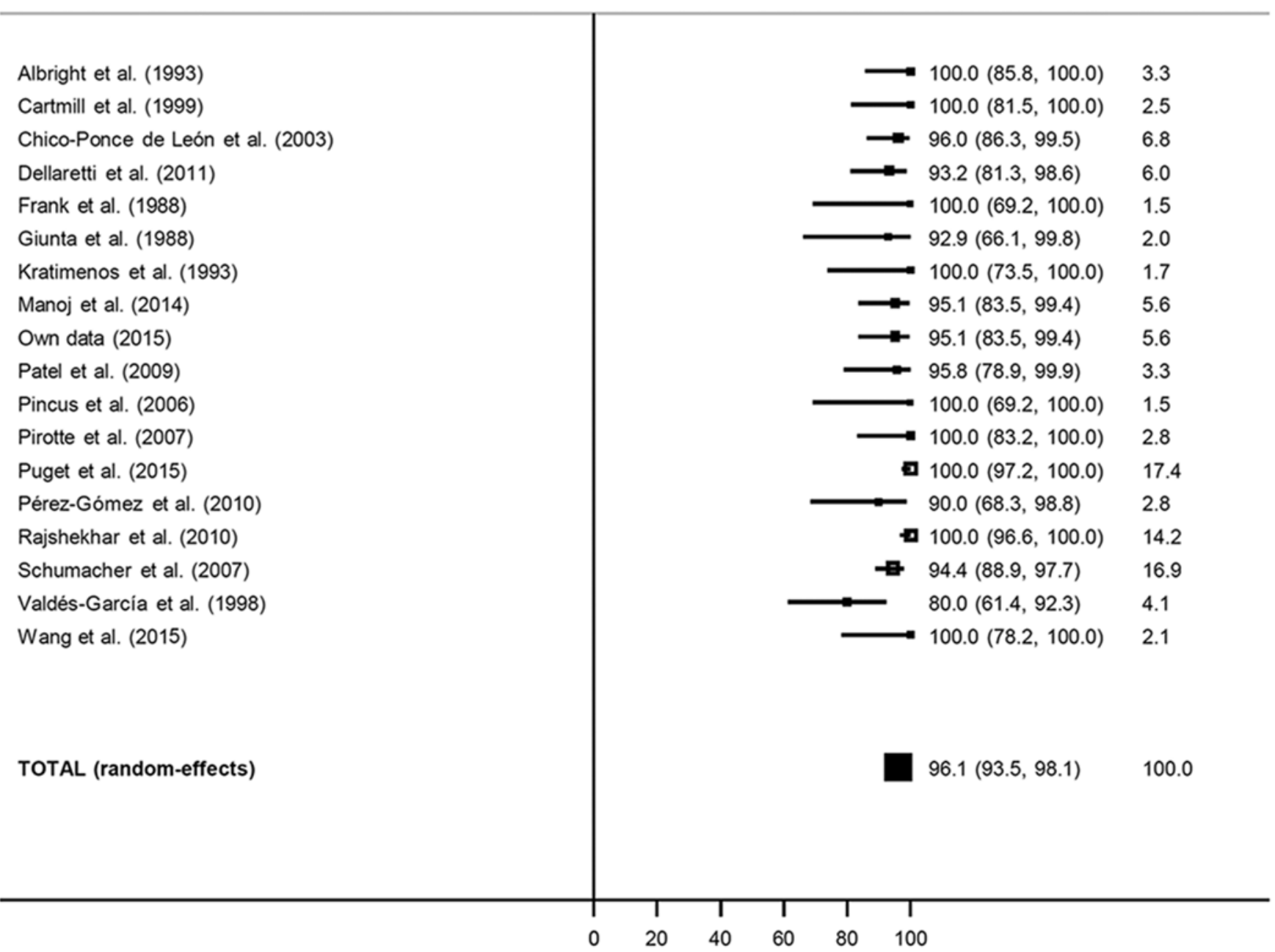

FIG. 2. Forest plot of diagnostic success, as assessed in the present meta-analysis. Squares and horizontal bars indicate point estimates and $95 \%$ confidence intervals of weighted proportions in the individual studies. The last line indicates the pooled proportions with $95 \%$ confidence intervals calculated with random-effects meta-analysis.

statistical software, release 12 (StataCorp LP). Statistical tests were 2-sided and used a significance level of $\mathrm{p}<$ 0.05 .

\section{Results}

\section{Search Results}

The search strategy retrieved 945 publications (Fig. 1). Screening of the titles and abstracts showed that 907 articles did not meet criteria for inclusion in the meta-analysis. Full text was retrieved for 38 studies. Review of these studies led to the elimination of 21 for not meeting inclusion criteria for the meta-analysis. Thus, including our own institutional series, a total of 18 studies were included in the present meta-analysis. . $, 5,6,8,9,11,16,17,19-23,25,29,32,33$

\section{Study Population}

The key characteristics of the patients and studies ana- lyzed are listed in Table 1. The studies comprised a sample of 735 patients. ${ }^{2,5,6,8,9,11,16,17,19-23,25,29,32,33}$ The cohorts varied between 10 and 130 patients, with a median of 24 patients per study. Annually, a median of 3.1 patients received stereotactic biopsy for BSTs in the individual institutions (range 1.4-10.0 patients per year). The chosen biopsy trajectory was transfrontal in $60 \%$ of patients and transcerebellar in $40 \%$. However, when comparing the biopsy trajectories at a study level it became evident that several institutions almost exclusively performed biopsies with only 1 of these trajectories (interquartile range [IQR] of $7 \%-88 \%$ for the transfrontal approach and $12 \%-93 \%$ for the transcerebellar approach).

\section{Meta-Analysis}

The weighted proportions for diagnostic success and procedure-related complications (overall and permanent morbidity and mortality) across the studies are shown in Figs. 2-5. In detail, the weighted average proportion 
Albright et al. (1993)

Cartmill et al. (1999)

Chico-Ponce de León et al. (2003)

Dellaretti et al. (2011)

Frank et al. (1988)

Giunta et al. (1988)

Kratimenos et al. (1993)

Manoj et al. (2014)

Own data (2015)

Patel et al. (2009)

Pincus et al. (2006)

Pirotte et al. (2007)

Puget et al. (2015)

Pérez-Gómez et al. (2010)

Rajshekhar et al. (2010)

Schumacher et al. (2007)

Valdés-Garcia et al. (1998)

Wang et al. (2015)

10)

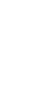

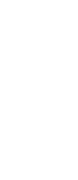

Weight, \%

(random)

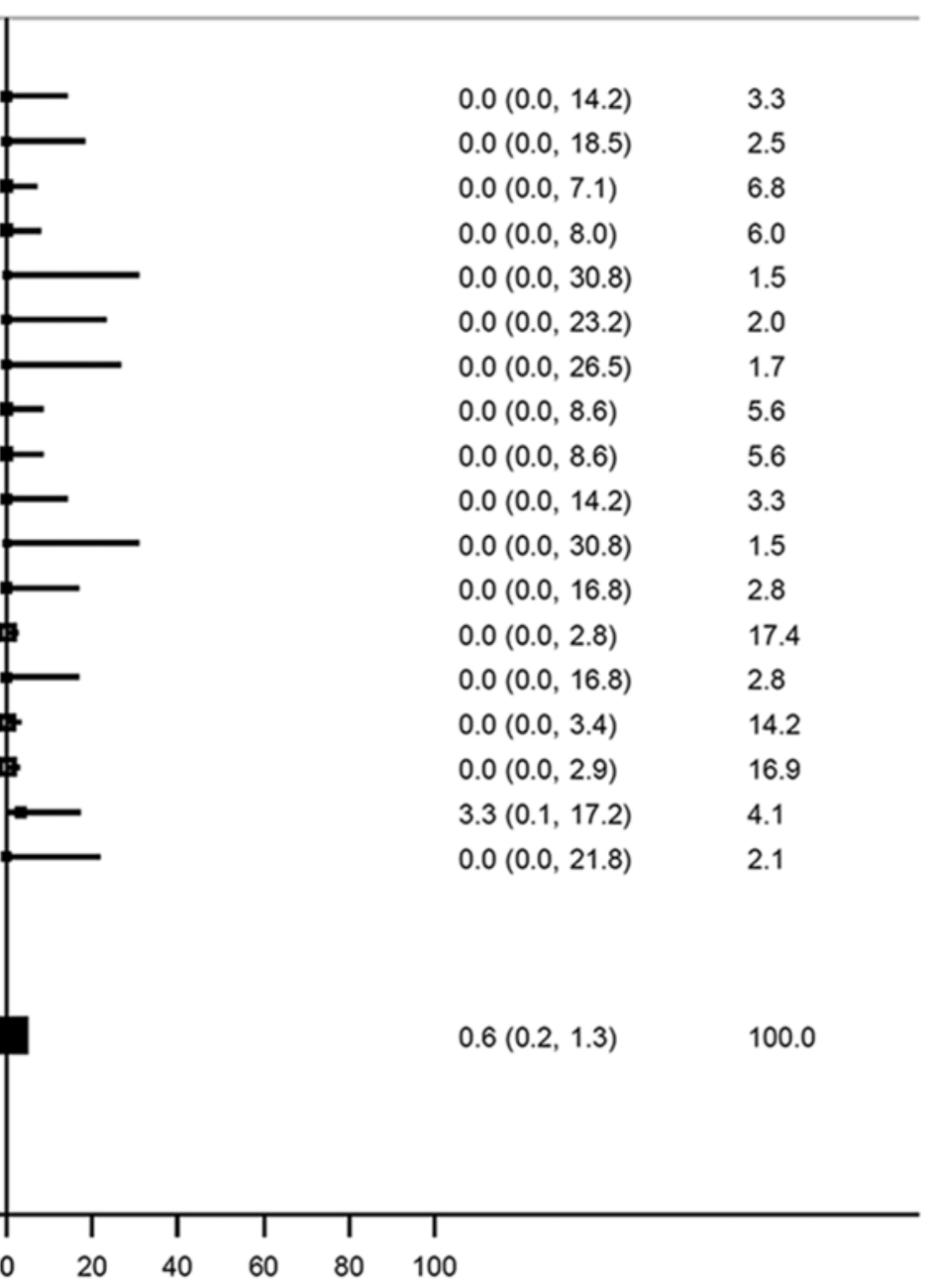

FIG. 3. Forest plot of mortality, as assessed in the present meta-analysis. Squares and horizontal bars indicate point estimates and $95 \%$ confidence intervals of weighted proportions in the individual studies. The last line indicates the pooled proportions with $95 \%$ confidence intervals calculated with random-effects meta-analysis.

calculated by random-effects modeling was $96.1 \%(95 \%$ CI $93.5 \%-98.1 \%)$ for diagnostic success, $6.7 \%$ (95\% CI 4.2\%-9.6\%) for overall morbidity, $0.6 \%$ (95\% CI $0.2 \%-$ $1.4 \%$ ) for permanent morbidity, and $0.6 \%$ (95\% CI $0.2 \%-$ $1.3 \%$ ) for mortality. Death was reported for only 1 patient within the analyzed cohort and was attributed to development of progressive brainstem edema. ${ }^{32}$

Sensitivity analysis for random-effects models that calculated pooled proportions upon exclusion of single studies in turn showed similar results, indicating that overall estimates were not driven by the findings of single studies (Supplementary Fig. 1). Between-study heterogeneity was absent for mortality $\left(\mathrm{I}^{2}=0.0 \%, 95 \%\right.$ CI $\left.0.0 \%-50.0 \%\right)$ and permanent morbidity $\left(\mathrm{I}^{2}=0.0 \%, 95 \%\right.$ CI $\left.0.0 \%-53.6 \%\right)$, mild for overall morbidity $\left(\mathrm{I}^{2}=48.4 \%\right.$, 95\% CI $9.5 \%-$ $70.6 \%)$, and moderate for diagnostic success $\left(\mathrm{I}^{2}=56.9 \%\right.$, 95\% CI 27.1\%-74.5\%) (Fig. 2-5). Funnel plots of studyspecific proportions were approximately symmetrical, and most data points were within the funnel area, indicating low evidence for presence of publication bias (Supplementary Fig. 2).

\section{Subgroup Analyses}

Meta-regression for subgroup analysis at the study level identified no significant correlation between the analyzed outcome measures and the distribution of chosen biopsy trajectories (transfrontal vs transcerebellar), distribution of the mean patient age in the individual studies, the year of publication, or the number of biopsy procedures performed annually in each center (Supplementary Table 2).

\section{Pathological Results}

A detailed list of the pathological entities diagnosed throughout the studies included in the present meta-analysis is shown in Table 2. In brief, histopathological evaluation revealed brain tumors in $92.0 \%$ of brainstem lesions. 
Albright et al. (1993)

Cartmill et al. (1999)

Chico-Ponce de León et al. (2003)

Dellaretti et al. (2011)

Giunta et al. (1988)

Kratimenos et al. (1993)

Manoj et al. (2014)

Own data (2015)

Patel et al. (2009)

Pincus et al. (2006)

Pirotte et al. (2007)

Puget et al. (2015)

Pérez-Gómez et al. (2010)

Rajshekhar et al. (2010)

Schumacher et al. (2007)

Valdés-Garcia et al. (1998)

Wang et al. (2015)

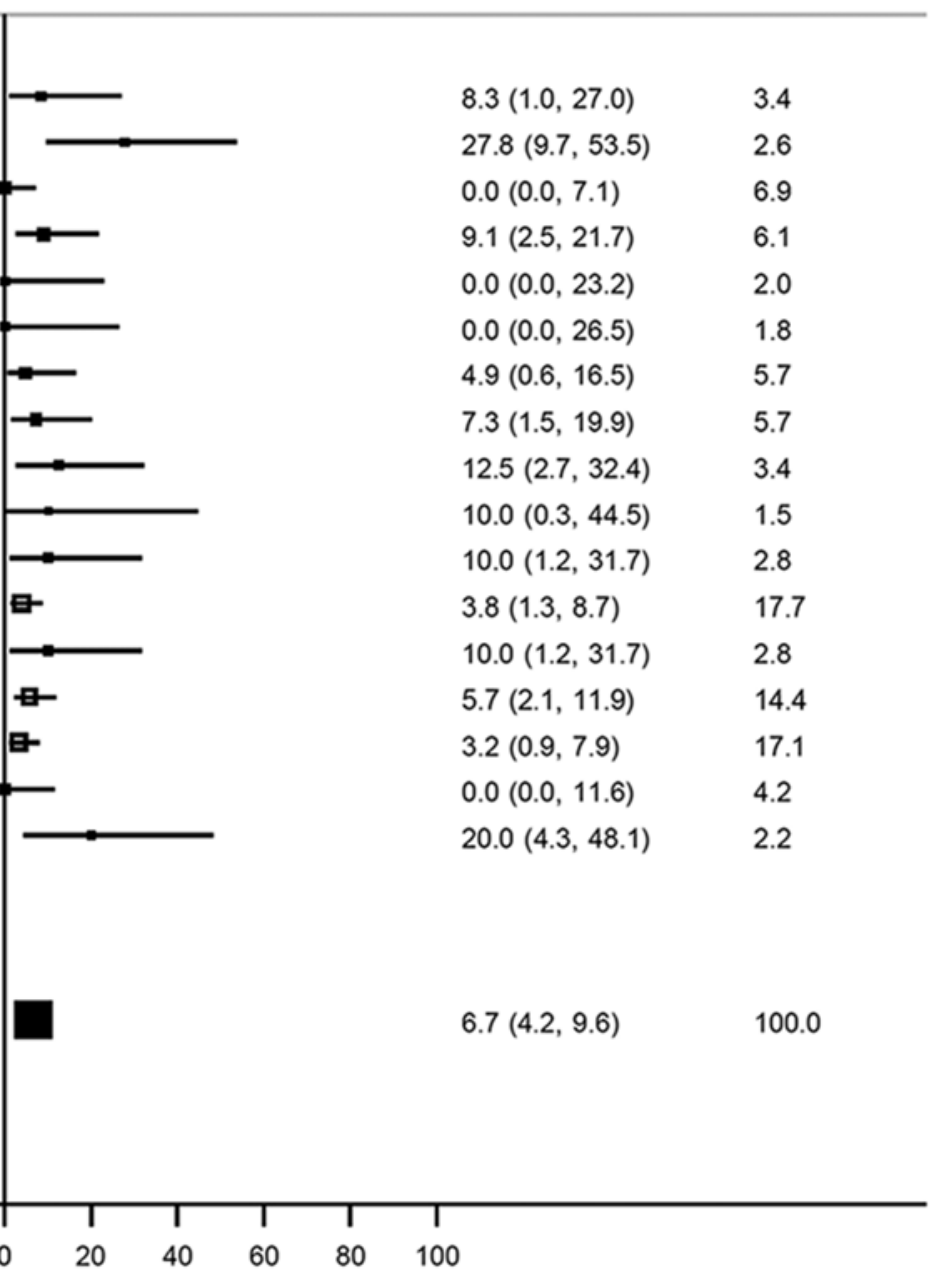

TOTAL (random-effects)

$\begin{array}{ll}8.3(1.0,27.0) & 3.4 \\ 27.8(9.7,53.5) & 2.6 \\ 0.0(0.0,7.1) & 6.9 \\ 9.1(2.5,21.7) & 6.1 \\ 0.0(0.0,23.2) & 2.0 \\ 0.0(0.0,26.5) & 1.8 \\ 4.9(0.6,16.5) & 5.7 \\ 7.3(1.5,19.9) & 5.7 \\ 12.5(2.7,32.4) & 3.4 \\ 10.0(0.3,44.5) & 1.5 \\ 10.0(1.2,31.7) & 2.8 \\ 3.8(1.3,8.7) & 17.7 \\ 10.0(1.2,31.7) & 2.8 \\ 5.7(2.1,11.9) & 14.4 \\ 3.2(0.9,7.9) & 17.1 \\ 0.0(0.0,11.6) & 4.2 \\ 20.0(4.3,48.1) & 2.2\end{array}$

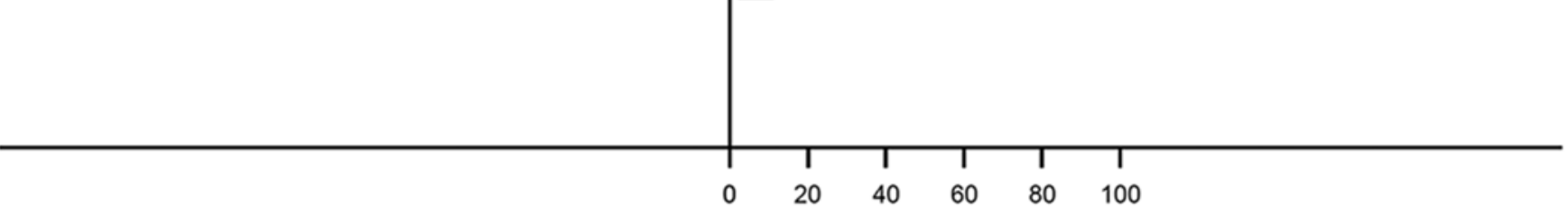

FIG. 4. Forest plot of overall morbidity, as assessed in the present meta-analysis. Squares and horizontal bars indicate point estimates and 95\% confidence intervals of weighted proportions in the individual studies. The last line indicates the pooled proportions with $95 \%$ confidence intervals calculated with random-effects meta-analysis.

Glial neoplasms were found in $84.4 \%$ of biopsy samples, primitive neuroectodermal tumors in $3.0 \%$, and ependymomas in $2.2 \%$. Nonneoplastic lesions-most commonly originating from infectious disease-accounted for $4.5 \%$ of biopsy samples. Stereotactic biopsy was nondiagnostic in the remaining $3.5 \%$ of patients.

\section{Discussion}

Our meta-analysis of 735 patients with pedBSTs indicates that stereotactic biopsy is a safe diagnostic procedure and is associated with a low rate of procedure-related complications (overall morbidity $6.7 \%$, permanent morbidity $0.6 \%$, mortality $0.6 \%$ ). Furthermore, it allows adequate tissue sampling in $96.1 \%$ of cases, which is a prerequisite for histological diagnosis as well as for the molecular characterization of these tumors.

Over the last decade, there has been an increasing understanding of the molecular, biological, and genetic make-up of pedBSTs in general and DIPGs specifically, which has revealed that these tumors are not a homogeneous entity, but rather comprise several distinct molecular subgroups, and that they are different from other pediatric gliomas and also different from adult gliomas., ${ }^{4,12,28}$ This strongly suggests that effective therapies for pediatric DIPGs may be distinct from effective therapies for other high-grade gliomas in pediatric or adult patients and highlights the problems of the past decades where clinical trials for pediatric DIPG were primarily chosen based on antitumor activity defined in adult high-grade gliomas. ${ }^{28}$ The latter is nowadays considered to be one of the reasons why no molecularly targeted agent has been shown to significantly improve survival in a clinical trial for pediatric DIPG. Given this demonstration of safety, along with the significant information obtained, the ability to perform molecular testing on small biopsy specimens, the identification of potentially druggable targets, and the heterogeneity of these tumors, stereotactic biopsy may become an important prerequisite for the molecular characterization 
Cartmill et al. (1999)

Chico-Ponce de León et al. (2003)

Giunta et al. (1988)

Kratimenos et al. (1993)

Manoj et al. (1988)

Own data (2015)

Patel et al. (200)

Pincus et al. (2006)

Pirotte et al. (2007)

Puget et al. (2015)

Pérez-Gómez et al. (2010)

Rajshekhar et al. (2010)

Schumacher et al. (2007)

Valdés-Garcia et al. (1998)

Wang et al. (2015)

TOTAL (random-effects)

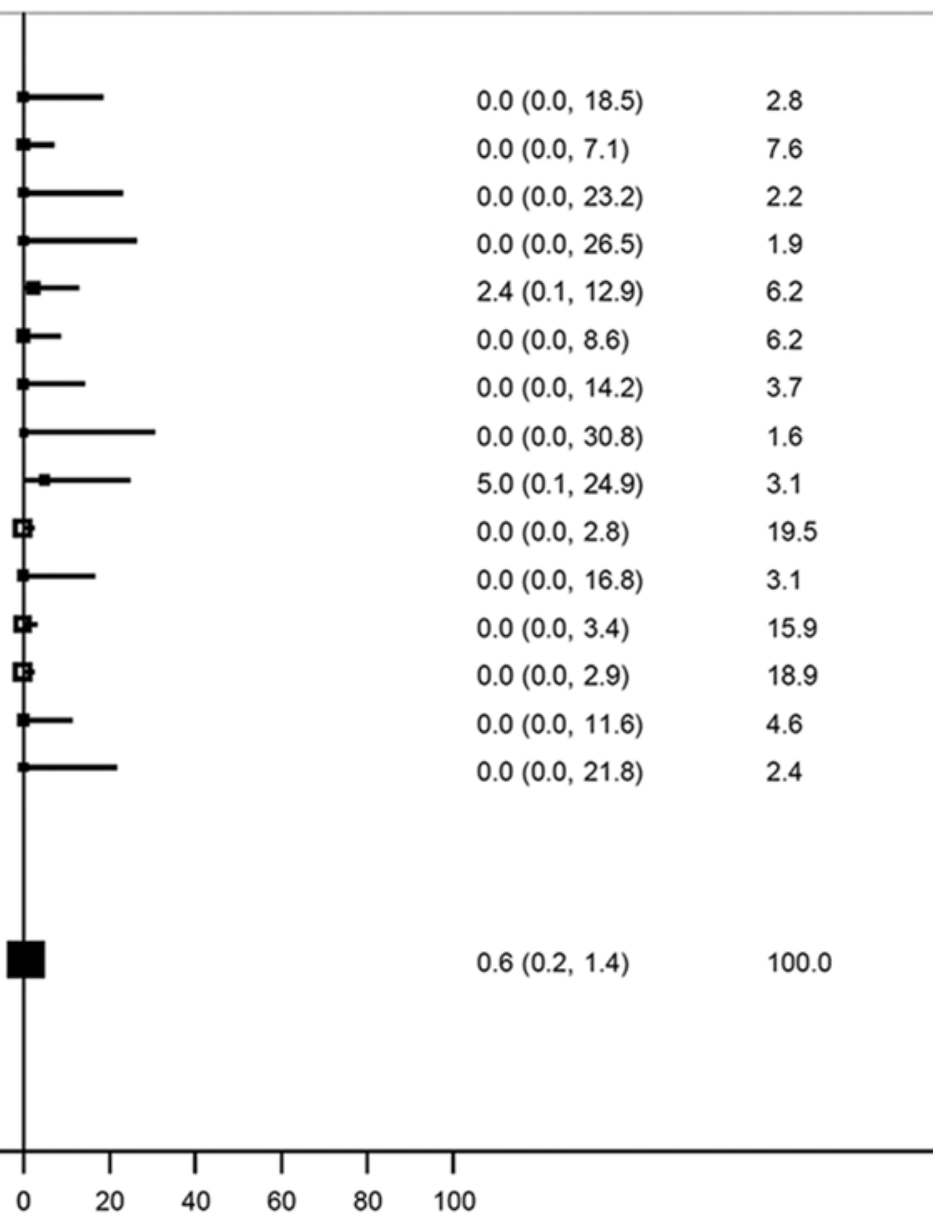

FIG. 5. Forest plot of permanent morbidity, as assessed in the present meta-analysis. Squares and horizontal bars indicate point estimates and $95 \%$ confidence intervals of weighted proportions in the individual studies. The last line indicates the pooled proportions with $95 \%$ confidence intervals calculated with random-effects meta-analysis.

of pedBSTs in general and DIPGs specifically as a crucial first step toward more individualized treatment concepts to improve the outcome for children harboring such lesions.

\section{References}

1. Abernathey CD, Camacho A, Kelly PJ: Stereotaxic suboccipital transcerebellar biopsy of pontine mass lesions. J Neurosurg 70:195-200, 1989

2. Albright AL, Packer RJ, Zimmerman R, Rorke LB, Boyett J, Hammond GD: Magnetic resonance scans should replace biopsies for the diagnosis of diffuse brain stem gliomas: a report from the Children's Cancer Group. Neurosurgery 33:1026-1030, 1993

3. Boviatsis EJ, Kouyialis AT, Stranjalis G, Korfias S, Sakas DE: CT-guided stereotactic biopsies of brain stem lesions: personal experience and literature review. Neurol Sci 24:97102,2003

4. Buczkowicz P, Hoeman C, Rakopoulos P, Pajovic S, Letourneau L, Dzamba M, et al: Genomic analysis of diffuse intrinsic pontine gliomas identifies three molecular subgroups and recurrent activating ACVR1 mutations. Nat Genet 46:451456,2014
5. Cartmill M, Punt J: Diffuse brain stem glioma. A review of stereotactic biopsies. Childs Nerv Syst 15:235-238, 1999

6. Chico-Ponce de León F, Perezpeña-Diazconti M, CastroSierra E, Guerrero-Jazo FJ, Gordillo-Domínguez LF, Gutiérrez-Guerra R, et al: Stereotactically-guided biopsies of brainstem tumors. Childs Nerv Syst 19:305-310, 2003

7. Dellaretti M, Reyns N, Touzet G, Dubois F, Gusmão S, Pereira JL, et al: Stereotactic biopsy for brainstem tumors: comparison of transcerebellar with transfrontal approach. Stereotact Funct Neurosurg 90:79-83, 2012

8. Dellaretti M, Touzet G, Reyns N, Dubois F, Gusmão S, Pereira JL, et al: Correlation among magnetic resonance imaging findings, prognostic factors for survival, and histological diagnosis of intrinsic brainstem lesions in children. $\mathbf{J}$ Neurosurg Pediatr 8:539-543, 2011

9. Frank F, Fabrizi AP, Frank-Ricci R, Spagnoli F, Frank G: Stereotactic brain biopsy in children. Childs Nerv Syst 4:191, 1988 (Abstract)

10. Freeman MF, Tukey JW: Transformations related to the angular and the square root. Ann Math Stat 21:607-611, 1950

11. Giunta F, Marini G, Grasso G, Zorzi F: Brain stem expansive lesions: stereotactic biopsy for a better therapeutic approach. Acta Neurochir Suppl (Wien) 42:182-186, 1988 
12. Grasso CS, Tang Y, Truffaux N, Berlow NE, Liu L, Debily MA, et al: Functionally defined therapeutic targets in diffuse intrinsic pontine glioma. Nat Med 21:555-559, 2015

13. Higgins JP, Thompson SG, Deeks JJ, Altman DG: Measuring inconsistency in meta-analyses. BMJ 327:557-560, 2003

14. Hood TW, Gebarski SS, McKeever PE, Venes JL: Stereotaxic biopsy of intrinsic lesions of the brain stem. J Neurosurg 65:172-176, 1986

15. Kickingereder P, Willeit P, Simon T, Ruge MI: Diagnostic value and safety of stereotactic biopsy for brainstem tumors: a systematic review and meta-analysis of 1480 cases. Neurosurgery 72:873-882, 2013

16. Kratimenos GP, Thomas DG: The role of image-directed biopsy in the diagnosis and management of brainstem lesions. Br J Neurosurg 7:155-164, 1993

17. Manoj N, Arivazhagan A, Bhat DI, Arvinda HR, Mahadevan A, Santosh V, et al: Stereotactic biopsy of brainstem lesions: Techniques, efficacy, safety, and disease variation between adults and children: A single institutional series and review. J Neurosci Rural Pract 5:32-39, 2014

18. Moher D, Liberati A, Tetzlaff J, Altman DG: Preferred reporting items for systematic reviews and meta-analyses: the PRISMA statement. PLoS Med 6:e1000097, 2009

19. Patel P, Balamurugan M: Transcerebellar stereotactic biopsy for brainstem lesions in children. J Pediatr Neurosci 4:1719, 2009

20. Pérez-Gómez JL, Rodríguez-Alvarez CA, Marhx-Bracho A, Rueda-Franco F: Stereotactic biopsy for brainstem tumors in pediatric patients. Childs Nerv Syst 26:29-34, 2010

21. Pincus DW, Richter EO, Yachnis AT, Bennett J, Bhatti MT, Smith A: Brainstem stereotactic biopsy sampling in children. J Neurosurg 104 (2 Suppl):108-114, 2006

22. Pirotte BJ, Lubansu A, Massager N, Wikler D, Goldman S, Levivier M: Results of positron emission tomography guidance and reassessment of the utility of and indications for stereotactic biopsy in children with infiltrative brainstem tumors. J Neurosurg 107 (5 Suppl):392-399, 2007

23. Puget S, Beccaria K, Blauwblomme T, Roujeau T, James S, Grill J, et al: Biopsy in a series of 130 pediatric diffuse intrinsic Pontine gliomas. Childs Nerv Syst 31:1773-1780, 2015

24. Rajshekhar V, Chandy MJ: Computerized tomography-guided stereotactic surgery for brainstem masses: a risk-benefit analysis in 71 patients. J Neurosurg 82:976-981, 1995

25. Rajshekhar V, Moorthy RK: Status of stereotactic biopsy in children with brain stem masses: insights from a series of 106 patients. Stereotact Funct Neurosurg 88:360-366, 2010

26. Recinos PF, Sciubba DM, Jallo GI: Brainstem tumors: where are we today? Pediatr Neurosurg 43:192-201, 2007

27. Ruge MI, Kickingereder P, Simon T, Treuer H, Sturm V: Stereotactic iodine-125 brachytherapy for treatment of inoperable focal brainstem gliomas of WHO grades I and II: feasibility and long-term outcome. J Neurooncol 109:273-283, 2012
28. Schroeder KM, Hoeman CM, Becher OJ: Children are not just little adults: recent advances in understanding of diffuse intrinsic pontine glioma biology. Pediatr Res 75:205-209, 2014

29. Schumacher M, Schulte-Mönting J, Stoeter P, Warmuth-Metz M, Solymosi L: Magnetic resonance imaging compared with biopsy in the diagnosis of brainstem diseases of childhood: a multicenter review. J Neurosurg 106 (2 Suppl):111-119, 2007

30. Shad A, Green A, Bojanic S, Aziz T: Awake stereotactic biopsy of brain stem lesions: technique and results. Acta Neurochir (Wien) 147:47-50, 2005

31. Steck J, Friedman WA: Stereotactic biopsy of brainstem mass lesions. Surg Neurol 43:563-568, 1995

32. Valdés-Gorcía J, Espinoza-Díaz DM, Paredes-Díaz E: Stereotactic biopsy of brain stem and posterior fossa lesions in children. Acta Neurochir (Wien) 140:899-903, 1998

33. Wang ZJ, Rao L, Bhambhani K, Miller K, Poulik J, Altinok $\mathrm{D}$, et al: Diffuse intrinsic pontine glioma biopsy: a single institution experience. Pediatr Blood Cancer 62:163-165, 2015

34. Warren KE: Diffuse intrinsic pontine glioma: poised for progress. Front Oncol 2:205, 2012

\section{Disclosures}

The authors report no conflict of interest concerning the materials or methods used in this study or the findings specified in this paper.

\section{Author Contributions}

Conception and design: Kickingereder, Ruge. Acquisition of data: Kickingereder, Hamisch, Fischer, Ruge. Analysis and interpretation of data: Kickingereder, Ruge. Drafting the article: all authors. Critically revising the article: all authors. Reviewed submitted version of manuscript: all authors. Approved the final version of the manuscript on behalf of all authors: Kickingereder. Statistical analysis: Kickingereder. Administrative/technical/material support: Kickingereder. Study supervision: Kickingereder, Ruge.

\section{Supplemental Information Online-Only Content}

Supplemental material is available with the online version of the article.

Supplementary Figures and Tables. https://thejns.org/doi/ suppl/10.3171/2017.2.PEDS1665.

\section{Correspondence}

Philipp Kickingereder, Department of Stereotactic and Functional Neurosurgery, University Clinics of Cologne, Kerpener Straße 62, Cologne 50937, Germany. email: philipp.kickingereder@gmail. com. 